\title{
PAndAS IN THE MIST: THE STELLAR AND GASEOUS MASS WITHIN THE HALOS OF M31 AND M33
}

\author{
Geraint F. Lewis ${ }^{1,2,14}$, Robert Braun ${ }^{3}$, Alan W. McConnachie ${ }^{4}$, Michael J. Irwin ${ }^{2}$, Rodrigo A. Ibata ${ }^{5}$, \\ Scott C. Chapman ${ }^{2}$, Annette M. N. Ferguson ${ }^{6}$, Nicolas F. Martin ${ }^{5}$, Mark Fardal $^{7}$, John Dubinski ${ }^{8}$, \\ Larry Widrow ${ }^{9}$, A. Dougal Mackey ${ }^{10}$, Arif Babul ${ }^{11}$, Nial R. Tanvir ${ }^{12}$, and Michael Rich ${ }^{13}$ \\ ${ }^{1}$ Sydney Institute for Astronomy, School of Physics A28, The University of Sydney, NSW 2006, Australia; geraint.lewis@ sydney.edu.au \\ ${ }^{2}$ Institute of Astronomy, University of Cambridge, Madingley Road, Cambridge CB3 OHA, UK \\ ${ }^{3}$ CSIRO Astronomy and Space Science, P.O. Box 76, Epping, NSW 1710, Australia \\ ${ }^{4}$ Dominion Astrophysical Observatory, 5071 West Saanich Road, Victoria, BC V9E 2E7, Canada \\ ${ }^{5}$ Observatoire de Strasbourg, 11, rue de l'Universite, F-67000 Strasbourg, France \\ ${ }^{6}$ Institute for Astronomy, University of Edinburgh, Blackford Hill, Edinburgh EH9 3HJ, UK \\ ${ }^{7}$ Department of Astronomy, University of Massachusetts, Amherst, MA 01003-9305, USA \\ ${ }^{8}$ Department of Astronomy \& Astrophysics, 50 St. George Street, University of Toronto, ON M5S 3H4, Canada \\ ${ }^{9}$ Department of Physics, Queen's University, 99 University Avenue, Kingston, ON K7L 3N6, Canada \\ ${ }^{10}$ Research School of Astronomy \& Astrophysics, Mount Stromlo Observatory, Cotter Road, Weston Creek, ACT 2611, Australia \\ ${ }^{11}$ Department of Physics \& Astronomy, University of Victoria, Victoria, BC V8W 3P6, Canada \\ 12 Department of Physics \& Astronomy, University of Leicester, Leicester LE1 7RH, UK \\ 13 Division of Astronomy, University of California, 8979 Math Sciences, Los Angeles, CA 90095-1562, USA \\ Received 2012 September 26; accepted 2012 November 16; published 2012 December 26
}

\begin{abstract}
Large-scale surveys of the prominent members of the Local Group have provided compelling evidence for the hierarchical formation of massive galaxies, revealing a wealth of substructure that is thought to be the debris from ancient and ongoing accretion events. In this paper, we compare two extant surveys of the M31-M33 subgroup of galaxies: the Pan-Andromeda Archaeological Survey of the stellar structure, and a combination of observations of the Hi gaseous content, detected at $21 \mathrm{~cm}$. Our key finding is a marked lack of spatial correlation between these two components on all scales, with only a few potential overlaps between stars and gas. The paucity of spatial correlation significantly restricts the analysis of kinematic correlations, although there does appear to be $\mathrm{H}$ I kinematically associated with the Giant Stellar Stream where it passes the disk of M31. These results demonstrate that different processes must significantly influence the dynamical evolution of the stellar and H I components of substructures, such as ram pressure driving gas away from a purely gravitational path. Detailed modeling of the offset between the stellar and gaseous substructures will provide a determination of the properties of the gaseous halos of M31 and M33.
\end{abstract}

Key words: dark matter - galaxies: abundances - galaxies: dwarf - galaxies: kinematics and dynamics Local Group

Online-only material: color figures

\section{INTRODUCTION}

In $\Lambda$ CDM cosmologies, galactic halos are built up over time through the accretion and cannibalization of smaller mass systems. Given the complexity of the dynamical interactions, these are studied in detail using computer simulations (e.g., Ibata \& Lewis 1998; Fardal et al. 2008), although, due to the difficulties in modeling baryonic physics, these typically only consider the evolution of dark matter. However, these predict that the outer region of galactic halos should be dominated by extensive tidal streams, whereas the shorter dynamical timescales of the inner halo result in complete destruction and a smooth stellar distribution (e.g., Bullock \& Johnston 2005; Cooper et al. 2010; Font et al. 2011).

The progenitors of substructure could possess a mix of stars and gas, generally more concentrated than the dark matter halos in which they reside. The precise composition of any infalling halo will depend upon its mass and evolutionary history, and we would expect dwarf galaxies to be stripped of their gas due to interactions within the Local Group, whereas larger galaxies will hold on to its gaseous component. While stars are effectively collisionless, more complex internal physics influences the

\footnotetext{
$\overline{{ }^{14} \text { Australian Research Council Future Fellow. }}$
}

evolution of gas, including internal shocking, cooling and collapse, star formation and its associated feedback, as well as ram-pressure stripping due to the presence of hot galactic halo gas (e.g., see Moore \& Davis 1994; Murakami \& Babul 1999; Bekki 2008; Mastropietro et al. 2009). This is quite apparent in the two major accretion events within the Milky Way halo, with the body and stream of the Sagittarius Dwarf Galaxy possessing no gas, having potentially lost it during its initial interaction with the Galaxy (Burton \& Lockman 1999, but see also Putman et al. 2004), while the Magellanic Stream appears to be completely devoid of stars, consisting of stripped gas, although the question of whether this is the result of tidal or ram-pressure forces has yet to be decided (Stanimirović et al. 2008; Besla et al. 2010; Diaz \& Bekki 2011). However, while we should expect different distributions for various disrupted components in M31 and M33, the complexities of ram-pressure stripping, which may require full magnetohydrodynamic approaches to simulate (Ruszkowski et al. 2012), make drawing robust conclusions difficult.

We are now in an era where large-scale surveys are providing a global picture of the formation and evolution of galaxies over cosmic time (e.g., see Abraham \& van den Bergh 2001). Given their distances, unraveling the fine details of galactic evolution is below the resolution achievable with the vast majority of 
galaxies in the universe. This is unfortunate, as it is on small scales, with the structure within the halos of large galaxies, that have presented the major challenge to the prevailing $\Lambda \mathrm{CDM}$ paradigm (e.g., the missing satellite problem; Klypin et al. 1999; Moore et al. 1999).

Luckily, the large members of the Local Group, the Milky Way, and the Andromeda (M31) and Triangulum (M33) galaxies are close enough for such fine-scale detail to be resolvable, although their immense angular scale presents a significant challenge to the observability of their extensive halos. To this end, the last decade has seen the advent of large, detailed surveys of both the stellar and gaseous components of Local Group members, for the first time providing a panoramic view of galaxy formation in action; for the Milky Way, this includes surveys such as RAVE (Siebert et al. 2011), SEGUE (de Jong et al. 2010), GASS (McClure-Griffiths et al. 2009), and others.

In this paper, we present a detailed comparison of the stellar and gaseous matter in the halos of our nearest large companions in the Local Group, M31 and M33, using a new map of stellar structure obtained through the Pan-Andromeda Archaeological Survey (PAndAS) and the most detailed maps of $\mathrm{H}$, obtained during several telescope campaigns. In Section 2, we outline the observational campaigns that resulted in the data presented, with a summary of their key scientific results. In Section 3, we compare the spatial and kinematic distributions of gaseous and stellar substructure. We present the interpretation of these correlations in Section 4 and our conclusions in Section 5.

\section{OBSERVATIONS AND PROPERTIES}

Some galaxies of the Local Group have been known since pre-history (e.g., see Section 3.1 of Geehan et al. 2006) ${ }^{15}$ and have played a pivotal role in our explorations of the workings of the universe. Reproducing a detailed account of this history is beyond the scope of this article, and the interested reader is directed to recent reviews (e.g., Tolstoy et al. 2009; Wyse 2010; Tolstoy 2011; McConnachie 2012). In the following we will focus upon the recent optical and radio surveys of M31 and M33, with a review of the observational programs and key scientific discoveries to date.

\subsection{Stellar Observations}

\subsubsection{Observational Program}

The PAndAS is a survey of $400 \mathrm{deg}^{2}$, covering the halo of M31 out to a distance of $150 \mathrm{kpc}$, and M33 to a corresponding distance of $50 \mathrm{kpc}$, undertaken as a large program on MegaCam, ${ }^{16}$ mounted on the $3.6 \mathrm{~m}$ Canada-France-Hawaii Telescope (CFHT). Integrations were sufficient to achieve photometric limits $g=25.5$ and $i=24.5$ at an $\mathrm{S} / \mathrm{N} \sim 10$, reaching several magnitudes below the tip of the red giant branch (RGB) at the distance of M31/M33 ( $D \sim 780-900 \mathrm{kpc}$; Conn et al. 2011). ${ }^{17}$ The photometric data taking was completed in early 2011, with the first published map, covering roughly half of the total observed area, presented in McConnachie et al. (2009),

\footnotetext{
15 Given its proximity, M31 is one of the few galaxies visible to the naked eye. Its existence was first documented by the Persian astronomer Abd al-Rahman Al-Sufi in his treatise on stellar astronomy titled "Kitab al-Kawatib al-Thabit al-Musawwar"(Book on the Constellations of the Fixed Stars), published in AD 964, where he both identified its position in the sky and summarized his observations.

16 http://cfht.hawaii.edu/Instruments/Imaging/Megacam

17 In the following, we adopt the distances $D_{M 31}=779 \mathrm{kpc}$ and $D_{M 33}=$ $820 \mathrm{kpc}$, each with an uncertainty of $\pm 20 \mathrm{kpc}$, as derived by Conn et al. (2012).
}

with the (almost) entire data set first presented in Richardson et al. (2011). The final map and high-level data products will be made publicly available in a forthcoming publication (McConnachie 2012; A. W. McConnachie et al. 2013, in preparation).

This study focuses upon RGB stars at the distance of M31 and M33, selected with cuts in color and magnitude (see Ibata et al. 2001, 2007; McConnachie et al. 2009). Figure 1 presents the distribution of RGB stars, overlain by a schematic map of the prominent stellar substructure; note that a nonlinear scaling has been applied to the RGB density, to bring out faint substructure, and contains a broad swath of metallicities to reveal metal-poor $(-3.0<[\mathrm{Fe} / \mathrm{H}]<-1.7)$, intermediate $(-1.7<[\mathrm{Fe} / \mathrm{H}]<$ $-0.7)$, and metal-rich $(-0.7<[\mathrm{Fe} / \mathrm{H}]<0.0)$ substructures (cf. Ibata et al. 2007). The thick solid line is the entire PAndAS footprint, with stellar substructure as labeled thin solid lines. The dashed curve represents a significant overdensity of globular clusters identified as the NW Group by Mackey et al. (2010). The large dot-dashed line corresponds to a circle of radius of $150 \mathrm{kpc}$ from the center of M31, whereas the smaller dot-dashed circle represents a distance of $50 \mathrm{kpc}$ from the center of M33. It is very apparent that accompanying M31 and M33 is a wealth of substructure consisting of extensive streams and dwarf galaxies (these will be discussed in more detail in Section 2.1.2, and see Richardson et al. 2011).

The stellar catalog derived from PAndAS is built upon earlier observations with the CFHT/MegaCam and CHFT/CFH12k (see McConnachie et al. 2003; Ibata et al. 2007). In parallel, a number of fields, targeting prominent substructure and dwarfs, were targeted with DEIMOS (Faber et al. 2003) on the $10 \mathrm{~m}$ Keck-II Telescope (e.g., Chapman et al. 2008; Collins et al. 2011). With a moderate resolution $(R \sim 6000)$, observations of 60-90 minutes around the prominent $\mathrm{CaT}$ absorption lines $\sim 8600 \AA$ resulted in an $\mathrm{S} / \mathrm{N} \sim 5$ for targets of $i \sim 21$, with a corresponding velocity resolution of $\sim 5-10 \mathrm{~km} \mathrm{~s}^{-1}$; these will be described in more detail in Section 3.2.

\subsubsection{Stellar Properties}

Detailed analysis of the stellar substructure in PAndAS will be presented in R. Ibata et al. (2013, in preparation) and A. W. McConnachie et al. (2013, in preparation), so here we focus on the key features.

There is a wealth of substructure apparent in the outer disk/inner halo regions of M31, revealed in the earlier WideField Camera on the $2.5 \mathrm{~m}$ Isaac Newton Telescope survey of the galaxy (Ibata et al. 2001; Richardson et al. 2008; see Figure 1), with the most significant structure being an extensive stellar stream, the Giant Stellar Stream (GSS), in the halo of M31. This is seen as a metal-rich substructure wrapped within a more metal-poor halo, and is apparently wrapped around the disk of M31 in the northeast (e.g., Ferguson et al. 2002; Ibata et al. 2007; Fardal et al. 2012). Accompanying the GSS is a series of streams, labeled B, C, and D, which lie perpendicular to the giant stream (Ibata et al. 2007), plus Stream A, a stellar overdensity $\sim 125 \mathrm{kpc}$ (in projection) from M31. Other features include the South West (SW) Cloud and the North West (NW) Stream (McConnachie et al. 2009; Mackey et al. 2010). As revealed in Figure 1, and as shown by Richardson et al. (2011), this latter feature appears to loop back toward M31 as the North East (NE) Stream and intercepts the And XXVII dwarf galaxy; following Carlberg et al. (2011), we identify this entire feature as the NW Stream, and label the two components of it as NW1 and NW2. Finally, also apparent in this image is another significant 


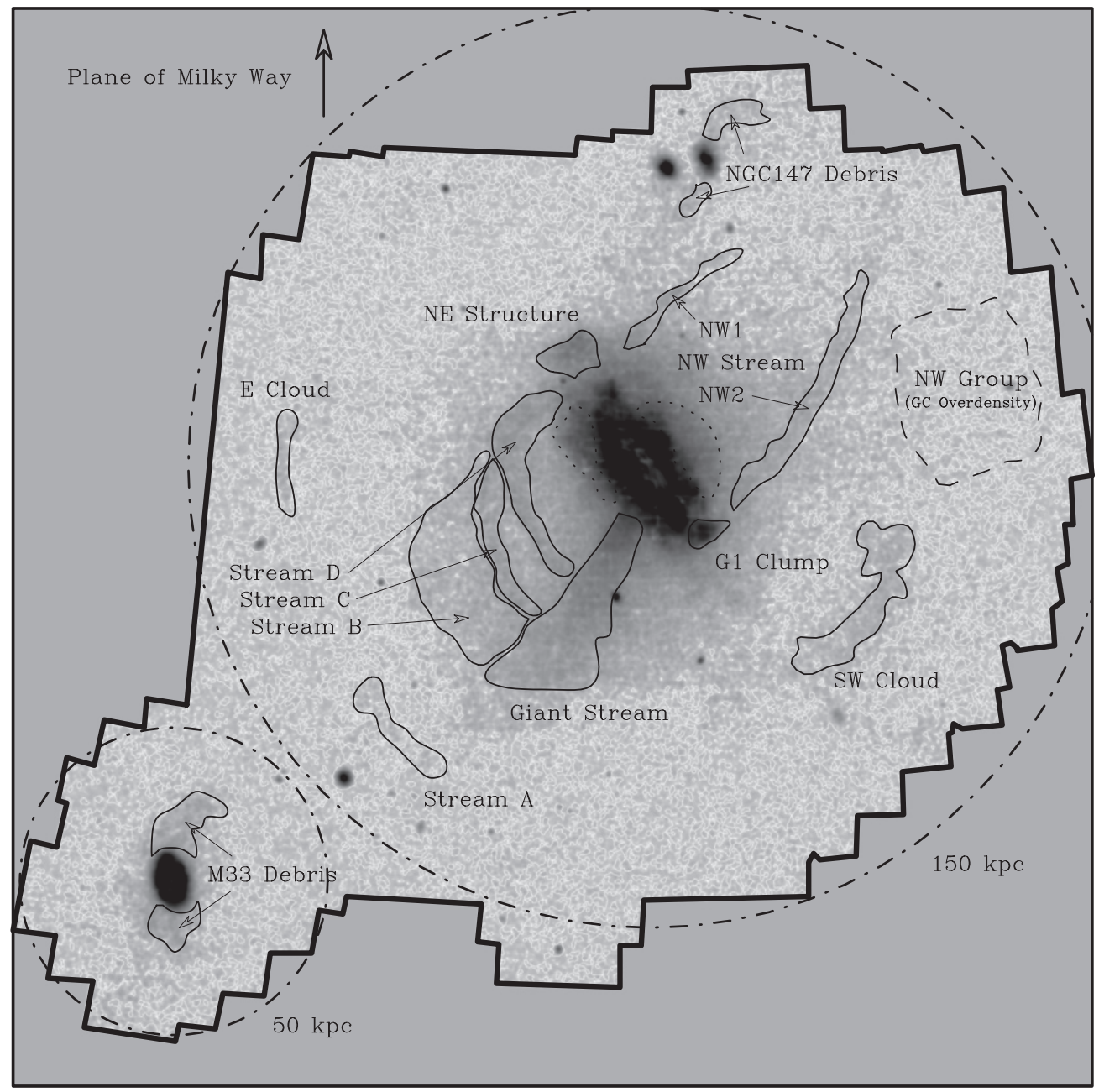

Figure 1. Schematic illustration of the prominent substructure apparent in red giant branch (RGB) stars; this encompasses a broad cut in metallicity and nonlinearly scales to enhance the stellar substructure. The thick solid line represents the PAndAS footprint, with the thinner solid lines resenting the main stellar substructure; as well as structure in the halo of M31, there is also tidal debris associated with M33 and NGC 147. A number of substructures have been presented in previous publications (see Section 2.1.2), but several additional features are apparent in this full map, including the Eastern Cloud and the broad swath of stars associated with Stream D (see also the PAndAS map presented in Richardson et al. 2011). The dashed line represents an overdensity of globular clusters identified in Mackey et al. (2010). Closer in to the disk of M31, the dotted lines represent the inner shells and loops thought to be wraps of the giant stellar stream. The large dot-dashed circle is at a radius of $150 \mathrm{kpc}$ from the center of $\mathrm{M} 31$, and the smaller being $50 \mathrm{kpc}$ from the center of $\mathrm{M} 33$.

overdensity of stars, named the Eastern (E) Cloud, located a distance of $\sim 125 \mathrm{kpc}$ from M31.

As well as the extensive substructure, the PAndAS observations revealed the presence of a smooth stellar halo of M31 out to $150 \mathrm{kpc}$ (Ibata et al. 2007; see also the SPLASH characterization of the smooth halo by Gilbert et al. 2012). Furthermore, the data reveal a wealth of dwarf galaxies (e.g., Martin et al. 2006, 2007; Richardson et al. 2011), globular clusters, and extended clusters (Huxor et al. 2008, 2011; Cockcroft et al. 2011; Tanvir et al. 2012); while these extended clusters (ECs) are more diffuse than normal globular clusters (Huxor et al. 2005), they appear to have very similar stellar populations (Mackey et al. $2006,2007)$ and do not appear to be dominated by dark matter (Collins et al. 2009).

The structure around M33, apparent as a distorted outer disk, was originally presented by McConnachie et al. (2009, 2010). This structure is quite clearly asymmetric about M33, being significantly more prominent in the north than in the south.

The extension of the PAndAS survey toward the north of M31, in the region of the two satellite galaxies, NGC 147 and NGC 185, reveals the presence of stellar debris that appears to have been torn from NGC 147, as well as a new dwarf galaxy, Cass II (also known as And XXX), which is a potential satellite of the 147/185 subgroup, and will be discussed in more detail in a forthcoming publication (M. J. Irwin et al. 2013, in preparation).

\subsection{H I Observations}

\subsubsection{Observational Program}

The extended environment of M31 and M33 was observed by Braun \& Thilker (2004) using the Westerbork Synthesis Radio Telescope (WSRT) as 14 single dish telescopes. A region of $60^{\circ} \times 30^{\circ}$ in R.A. $\times$ decl. was imaged in the $\mathrm{H}$ I emission line at an effective resolution of 49 arcmin with an rms sensitivity corresponding to a column density of $5 \times 10^{16} \mathrm{~cm}^{-2}$ over $\sim 30 \mathrm{~km} \mathrm{~s}^{-1}$. This unprecedented sensitivity permitted detection of $\mathrm{HI}_{\mathrm{I}}$ in emission from column densities that have previously only been probed by Ly $\alpha$ absorption toward background QSOs. This low-resolution survey has been supplemented by higher resolution total power observations made with the Green Bank Telescope (GBT). A region covering $7^{\circ} \times 7^{\circ}$ centered on M31 and $5^{\circ} \times 5^{\circ}$ centered on M33 was observed with GBT during 2002 October with multiple perpendicularly scanned coverages 


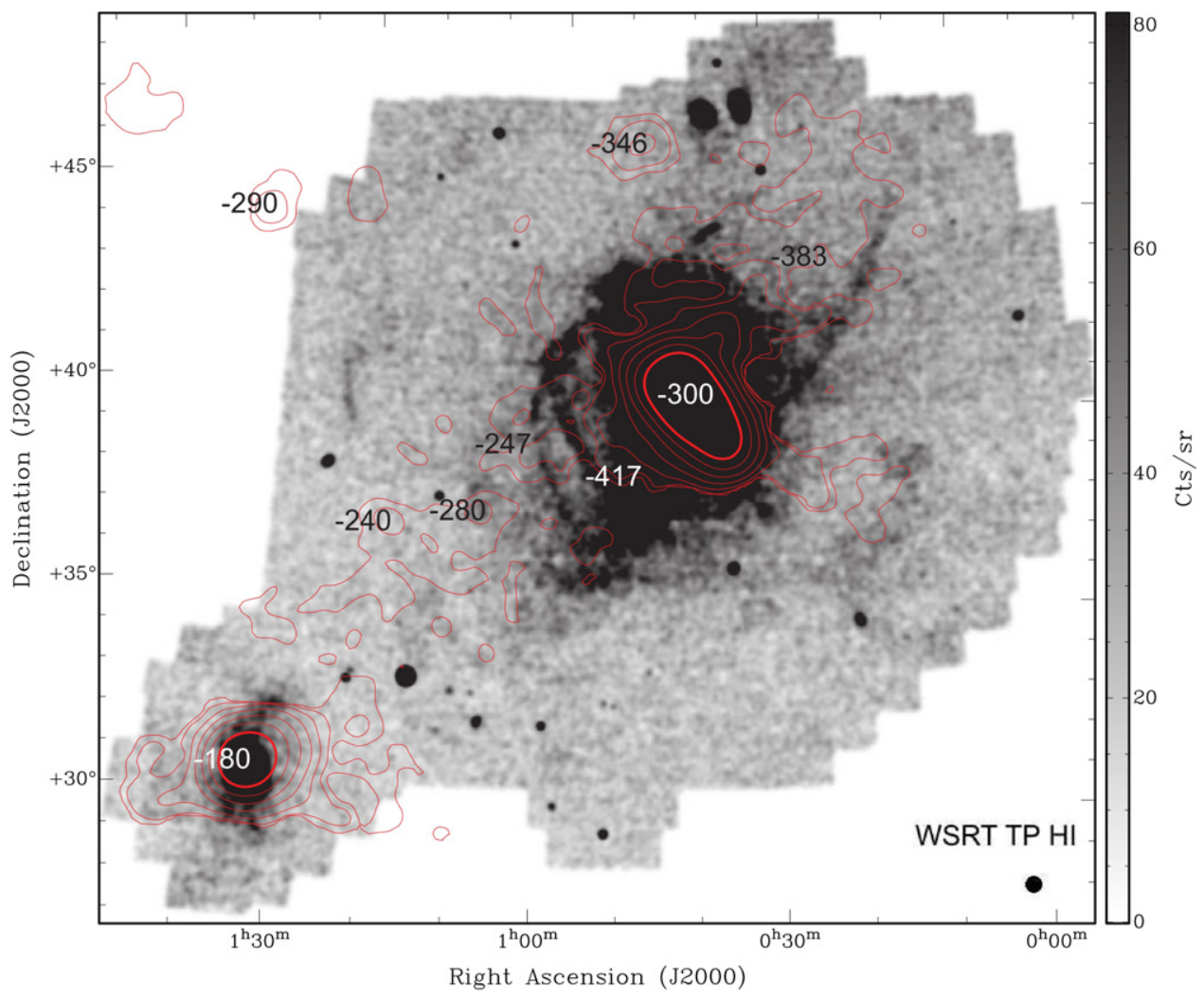

Figure 2. Distribution of H I gas (red contours), drawn from the survey of Braun \& Thilker (2004), overlaid on a surface density map of stars identified as being on the RGB within M31 and M33 in the entire PAndAS footprint (see McConnachie et al. 2009; Richardson et al. 2011). The logarithmic contours are drawn at integrated column densities of $\mathrm{HI}$, with $N_{\mathrm{HI}}=10^{17}-10^{20} \mathrm{~cm}^{-2}$ in steps of 0.5 dex, and the annotations indicate the heliocentric radial velocity of $\mathrm{HI}$ features. The stellar map again represents a broad range of metallicities and has a nonlinear stretch to accentuate the substructure.

(A color version of this figure is available in the online journal.)

yielding images with angular resolution as high as 9 arcmin. The M31 data have been presented previously by Thilker et al. (2004), while the M33 data were obtained with the same setup and have been reduced in a similar fashion. These moderate resolution data are supplemented with interferometric mosaic observations consisting of a 163 pointing WSRT coverage of M31 (Braun et al. 2009) and Very Large Array (VLA) coverage of M33 consisting of 6 pointings in the B and CS configurations and a 20 pointing mosaic in the D configuration. Some early M33 results based on the $\mathrm{B}$ and CS configuration data were presented in Thilker et al. (2002) while an independent reduction of all three VLA configurations has been presented by Gratier et al. (2010). The interferometric data provides resolution as high as 15 arcsec in M31 and as high as 5 arcsec in M33.

\subsubsection{H I Properties}

The Hi distribution in the extended M31 and M33 environment is overlain as contours on the surface density of RGB stars in the PAndAS survey in Figure 2. The H I contours delineate the diffuse gaseous filament that connects M31 and M33 as well as other filamentary features extending both to the northwest of the M31 disk and to the southwest. Heliocentric radial velocities of various features are indicated with the annotations. The intrinsically diffuse nature of these features has been verified with follow-up observations using the GBT directed at the brightest positions along the M31-M33 filament (Braun \& Thilker 2004). Despite employing a 25 times smaller beam area in these GBT observations, the features were detected at the same low column densities of only a few times $10^{17} \mathrm{~cm}^{-2}$. This rules out the possibility that the filament is simply a collection of unresolved clumps in the discovery observations. Several discrete features are also seen in the northern portion of the field. It is noteworthy that the M31-M33 filament connects the systemic heliocentric velocities of M31 $\left(\sim-300 \mathrm{~km} \mathrm{~s}^{-1}\right)$ and M33 $\left(\sim-180 \mathrm{~km} \mathrm{~s}^{-1}\right)$.

The more immediate $\mathrm{H}$ I environment of M31 is illustrated in Figure 3 where the GBT image (left panel) and WSRT mosaic (right panel) are similarly overlain on the PAndAS survey. At these higher angular resolutions, of 18 and 4 arcmin respectively, much of the diffuse gas is not detected but rather only the discrete High Velocity Cloud (HVC) features within about $100 \mathrm{kpc}$ of the disk. Of note is that the radial velocity of discrete HVC features follows the basic pattern of disk rotation with the most negative velocities occurring in the southwest and most positive in the northeast.

The immediate gaseous environment of M33 is illustrated in Figure 4 where the GBT image at 9 arcmin resolution (left panel) and the VLA mosaic with 2 arcmin resolution (right panel) are overlain on the PAndAS survey. M33 is not as rich in discrete HVC features as M31 but rather displays a very strong clumpy warp of the outer H I disk oriented toward the northwest and southeast, strongly suggestive of accretion fueling of the M33 disk by fall back from the M31-M33 filament.

\section{CORRELATIONS}

\subsection{Spatial Correlations}

\subsubsection{The Disk and Halo of M31}

First, we consider the large-scale distribution of RGB stars and $\mathrm{H}$ I gas presented in Figure 2, focusing upon material in the 


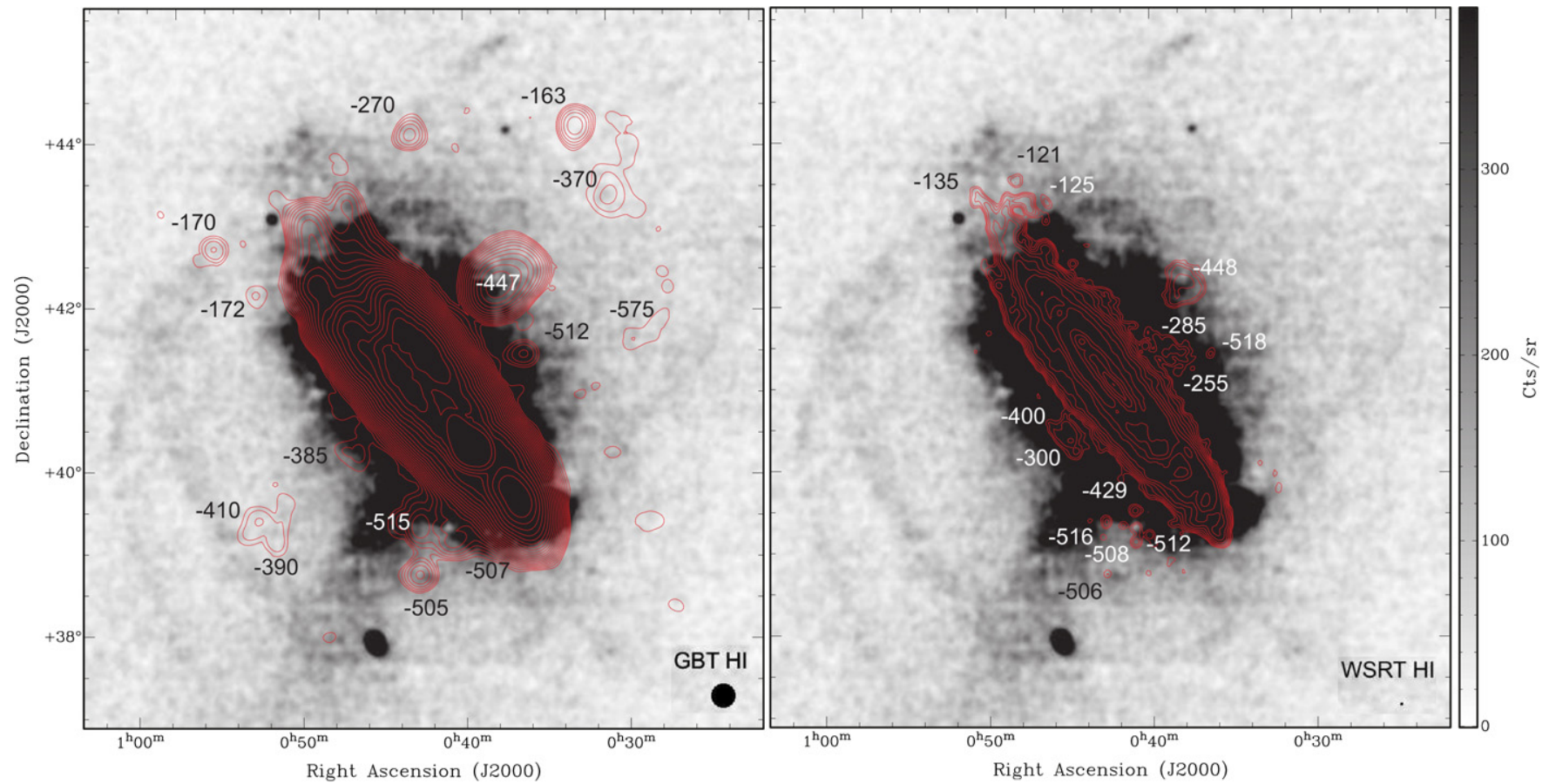

Figure 3. Same as Figure 2, but focused upon the main disk and inner halo substructure of M31. The left panel is overlain with H I contours drawn from the study of Thilker et al. (2004) which reveal structure at a spatial resolution of 18 arcmin. The logarithmic contours are drawn at peak brightness temperatures $T_{B}=0.025-20 \mathrm{~K}$ in steps of 0.15 dex. The right panel is overlain with the H I observations of Braun et al. (2009), further revealing smaller scale structure, at 4 arcmin resolution, in the gaseous component. The logarithmic contours are drawn at integrated column densities $N_{\mathrm{H}_{\mathrm{I}}}=7 \times 10^{18}$ to $4 \times 10^{21} \mathrm{~cm}^{-2}$ in steps of 0.28 dex. The annotations on both panels indicate the heliocentric radial velocity of $\mathrm{H}$ f features, and the image is $108 \mathrm{kpc}$ across.

(A color version of this figure is available in the online journal.)
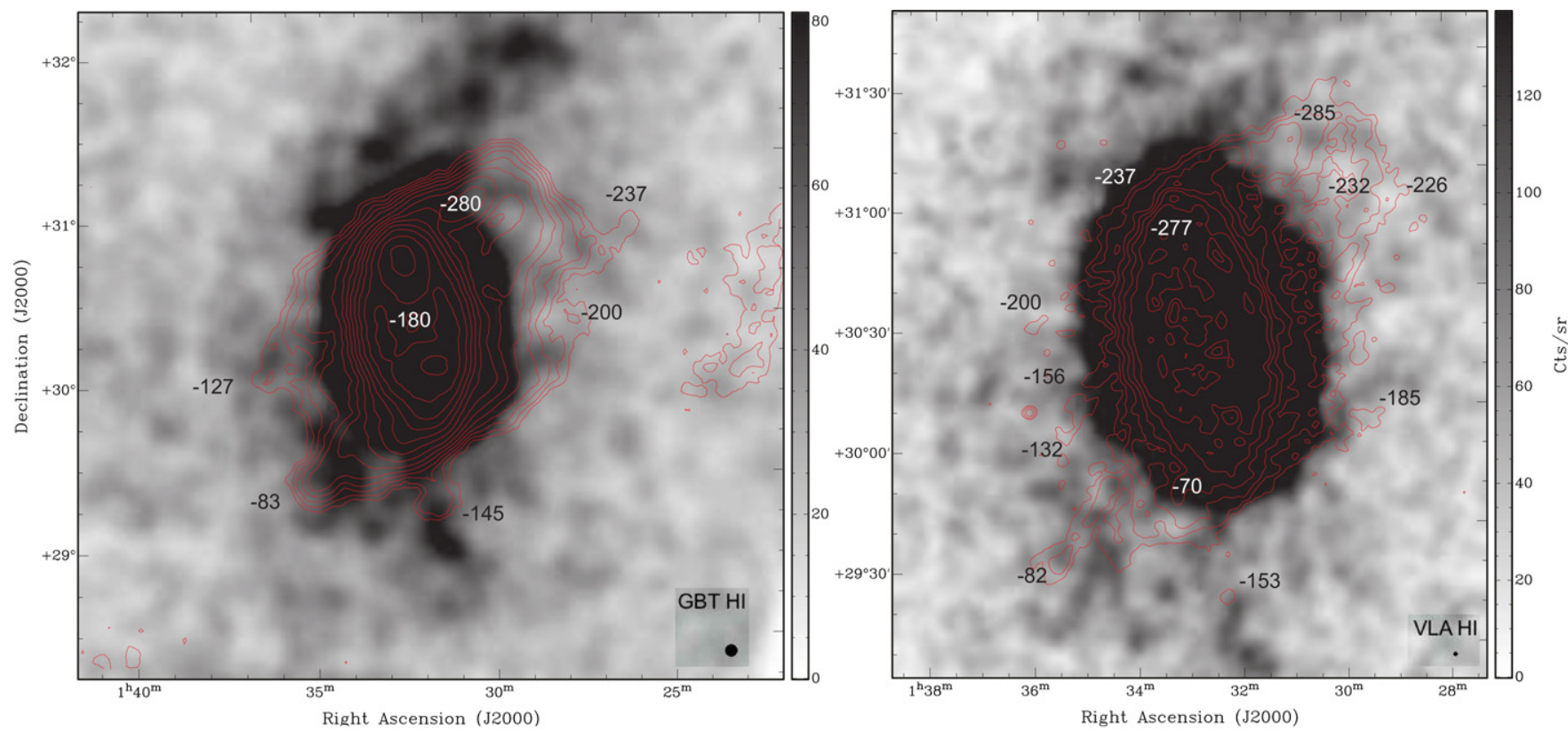

Figure 4. RGB stellar map of M33 and its associated tidal structure, overlain with the H i observations of Thilker et al. (2004) with the GBT, with a resolution of 9 arcmin (left panel), with logarithmic contours drawn at peak brightness temperatures $T_{B}=0.15-20 \mathrm{~K}$ in steps of 0.18 dex. The right panel presents the VLA observations of $\mathrm{H}$ I by Thilker et al. (2002) at 2 arcmin resolution. The logarithmic contours are drawn at peak brightness temperatures $T_{B}=0.9-30 \mathrm{~K}$ in steps of 0.26 dex. The annotations indicate the heliocentric radial velocity of $\mathrm{H}$ I features, and the image on the left is $60 \mathrm{kpc}$ across, whereas the image on the right is $35 \mathrm{kpc}$ across. (A color version of this figure is available in the online journal.)

vicinity of M31. Leaving aside the inner disk region, which will be examined in detail shortly, it is useful to consider material along the NW axis, connecting M31 and M33, and that which lies off this axis in the halo of M31.
It is clear that the eastern portion of the halo possesses one substantial stellar substructure, namely the Eastern Cloud, as well as a number of dwarf galaxies (see Richardson et al. 2011). This section of the halo is effectively devoid of a substantial 
quantity of $\mathrm{H}$ I material, except for a lone clump located to the far north, and some lower level material closer to the disk of M31, with no significant enhancement in the vicinity of any of the dwarf galaxies. Hence, there appears to be no substantive correlation between the stellar and gaseous material in this area of the halo.

The situation is very similar in the western halo, with one extensive stellar substructure, the SW Cloud, and a population of dwarf galaxies, and some potential, but low level, substructure features. Note that Mackey et al. (2010) also identified a potential overdensity of globular clusters, the NW Group, which is not associated with an overdensity in the stellar density (see Figure 1). Again, this area is almost devoid of significant $\mathrm{H}$ I detections, other than a large spur at $\sim 10^{\circ}$ to the major axis, extending $\sim 65 \mathrm{kpc}$ from the center of M31. Again, there is apparently little stellar substructure along the length of this gaseous spur, except at its tip where it overlies a portion of the SW Cloud. The physical implications of this association will be discussed in Section 4.

The most conspicuous stellar and gaseous substructures lie along the NW axis, along the line joining M31 and M33. In the region south of M31, there is significant stellar substructure in the form of the GSS, perpendicular steams, B, C, and D, close to the outer disk of M31, and the distant stream A. The gas in this region is significantly more extended, lying all along the axis. There is no significant overlap of H I with the GSS, with the gaseous material extending from the disk of M31 over the inner stream fields and toward M33. Significant H I also extends northward from the disk of M31, over the region encompassing the NW stream, although the gaseous material is more extensively spread than the stellar substructure in this area.

The two panels in Figure 3 zoom into the outer disk region of M31, overlain with $\mathrm{HI}$ at the two resolutions discussed earlier. While the GSS and Stream C are apparent as stellar substructures, with Stream B stretching back toward M31, there appears to be no significant correlation between the stellar material and H I gas. Intriguingly, there is some Hi emission, apparent as population of distinct clouds, aligned with the GSS, but offset by $\sim 15 \mathrm{kpc}$; as will be discussed in Section 3.2.1, this emission is also kinematically correlated with the GSS (see Braun et al. 2009).

Clearly, the $\mathrm{H}$ I associated with the stellar disk possesses similar distortions to the underlying stars, including a northern spur, located in the outer disk of M31 (close to the NE Structure in Figure 1, see also Figure 1 in Richardson et al. 2008) and a potential overdensity near the G1 clump; however, it is difficult to discern whether either the stellar or gaseous material has recently been accreted or is actually disk material that has been tidally distorted due to interactions (Ibata et al. 2005; Faria et al. 2007).

\subsubsection{The Disk and Halo of M33}

At the resolution provided by the Braun \& Thilker (2004; Figure 2), the $\mathrm{H} \mathrm{I}$ emission from M33 is seen to be quite extensive and orientated E-W across the galaxy. The stellar substructure, on the other hand, lies mainly on the NW axis, joining the disk of M33 at the northern tip of the galaxy.

The situation becomes clearer in Figure 4, which focuses upon the stellar distribution of M33 overlain with $\mathrm{H}$ I emission seen with the GBT (left panel) and VLA (right panel; Thilker et al. 2002, 2004). The striking feature in both the H I and stellar material is the substantial stream pointing toward the
NW, as well as a less pronounced component in the SE; this extended H I emission was discussed by Putman et al. (2009), with the conclusion that it results from the tidal interaction between M33 and M31. A similar conclusion with regards to the stellar component was reached by McConnachie et al. (2009), also presenting a numerical model for the tidal interaction of the two galaxies which results in a consistent stellar feature. However, while the stellar and gaseous material possess a similar alignment, they are distinct spatially, with the majority of the gas streaming from the western edge of M33, whereas the stellar material streams from the northern tip of the galaxy. Furthermore, while both the northern and southern stellar and gaseous components are asymmetrical, it is apparent that the stellar component possesses a much more pronounced asymmetry.

\subsection{Kinematic Correlations}

While the radio observations provide a global picture of velocities, the nature of multi-object optical spectroscopy ensures that the stellar kinematics are determined in a series of discrete fields. As well as the parallel kinematic survey undertaken by members of the PAndAS team (e.g., Ibata et al. 2004), significant effort has been undertaken by other groups (e.g., the SPLASH survey; Kalirai et al. 2009, 2010; Gilbert et al. 2009). However, the general lack of distinct spatial correlations (see Section 3.1) means that direct comparison of kinematics at specific locations is not possible, and only a general comparison can be undertaken.

\subsubsection{The Disk and Halo of M31}

The most prominent stellar feature in the halo of M31 is the GSS, lying close to the (three-dimensional) distance where it meets the spiral disk, sweeping backward to over $\sim 100 \mathrm{kpc}$ behind (Ibata et al. 2001; McConnachie et al. 2003; Fardal et al. 2012). A stellar kinematic survey of the GSS shows a strong velocity gradient (Ibata et al. 2004); the most distant tip of the stream, 4.5 away from M31's disk, has velocities of $v_{h}{ }^{18} \sim-300 \mathrm{~km} \mathrm{~s}^{-1}$, placing it essentially at rest with regards to M31, while those approaching the disk are traveling at $v_{h} \sim-500 \mathrm{~km} \mathrm{~s}^{-1}\left(v_{\mathrm{M} 31} \sim-200 \mathrm{~km} \mathrm{~s}^{-1}\right)$. While there is no apparent gas associated with the extent of the GSS (Section 3.1), there is significant parallel $\mathrm{HI}_{\mathrm{I}}$ emission. This gas possesses a strong velocity gradient, but different to that of the GSS (Figure 2); close to the M31 disk, the gas is moving close to systemic velocity of M31, whereas away from the disk, the velocity becomes more positive. The velocity gradient bridges the systemic velocities of M31 and M33, demonstrating a direct connection and common origin.

North of M31, along the M31-M33 axis, there is significant H I emission which seemingly overlaps with the NW Stream. The velocity of the $\mathrm{HI}\left(v_{h} \sim-380 \mathrm{~km} \mathrm{~s}^{-1}\right)$ appears to be a continuation of the gaseous stream connecting the two galaxies. Presently, there are no velocities of the stellar structure in this region, and so it is difficult to comment on any putative connection, although the numerical models suggest that these features are unrelated (Bekki 2008; McConnachie et al. 2009).

Figure 3 reveals the kinematic structure of the $\mathrm{H} I$ in the outer disk and inner halo of M31, where there is an enhancement of $\mathrm{H}$ I close to, but not completely aligned with, the GSS where it meets the disk of M31 (Section 3.1.1). Intriguingly, while offset

\footnotetext{
18 In the following, $v_{h}$ refers to heliocentric velocities, whereas $v_{\mathrm{M} 31}$ is relative to $\mathrm{M} 31$.
} 
spatially, this gas is moving at $v_{h} \sim-510 \mathrm{~km} \mathrm{~s}^{-1}$, similar to the velocity of the stellar content of the GSS, and with $v_{\mathrm{M} 31}$ $\sim-200 \mathrm{~km} \mathrm{~s}^{-1}$. At higher resolution, this gas decomposes into distinct clumps; this will be discussed in Section 4.

Also seen in stellar maps underlying Figure 3 are Streams $\mathrm{C}$ and D. While there is apparently no emission associated with Stream C, there is one blob of emission almost overlaying Stream D with $v_{h} \sim-400 \mathrm{~km} \mathrm{~s}^{-1}$. This emission arises close to two kinematic fields on Stream D obtained by Chapman et al. (2008), both of which yielded velocities of $-391 \mathrm{~km} \mathrm{~s}^{-1}$; given the similarity of these velocities, and their distinct difference from the large-scale $\mathrm{H}$ I gradient between M31 and M33 (with an expected velocity of $\sim-250 \mathrm{~km} \mathrm{~s}^{-1}$ ), this is strongly suggestive of an association between the stellar and gaseous material in Stream D.

\subsubsection{The Disk and Halo of M33}

There is a gross alignment of the stellar debris and that of the $\mathrm{H}$ I emission in the outskirts of M33, although again there is the spatial offset between the two components (Section 3.1 and Figure 4). The kinematic properties of the gaseous material within the disk of M33 can be described with a galactic rotation model (i.e., Corbelli 2003), and also the emission beyond the edge of the stellar disk. Unfortunately, the only stellar kinematics obtained beyond the optical disk were obtained along the southern major axis (McConnachie et al. 2006), away from the prominent stellar debris (McConnachie et al. 2009, 2010), and hence no detailed comparison can be made.

\section{INTERPRETATION}

What does the general lack of spatial and kinematic correlations between stellar substructure and $\mathrm{H}$ i tell us about the ongoing accretion in the M31-M33 system? Clearly, the axis connecting these two galaxies contains the majority of the substructure, but it appears that, in this case, the stellar and $\mathrm{H}$ I are the result of distinctly different accretion events; the main gaseous bridge through this region arises from the interaction between M31 and M33 alone, with any stellar material too sparsely distributed to be detected (Bekki 2008; McConnachie et al. 2009). Given M33's relatively large mass, and potentially large impact parameter, it has been able to retain the bulk of its stars and gas during the interaction with M31, although the interaction has distorted both the stellar and gaseous disk of M33.

The progenitor of the GSS, however, would have been a lower mass system that has been interacting with M31, and due to this interaction any progenitor gas was lost long ago, while the remnant stellar debris exists in the form of tidal streams and shell-like caustics. At first glance, the lack of significant gaseous structures corresponding to the GSS and other associated stellar features may seem surprising. However, as noted previously, while the gaseous and stellar components of an orbiting satellite are both subject to gravitational (tidal) forces, the gas is also subject to shocks and hydrodynamic drag. One possibility is that ram-pressure stripping led to nearly complete loss of gas from the GSS progenitor well before the formation of the observed GSS and associated structures. However, even if the ram pressure is not sufficiently efficient to completely strip the progenitor of its gas, hydrodynamic interactions can erase potential spatial and kinematic correlations between the two components. We are presently studying the gaseous and stellar streams using high-resolution numerical simulations
(M. Shannon et al., in preparation) and preliminary results suggest that the two components can experience significant dislocation over the course of a single orbit, with the loss of angular momentum and energy due to hydrodynamic forces causing the gas to sink to the center of M31, unlike the much longer-lived stellar structures.

One intriguing correlation occurs where the GSS meets the disk of M31 (see Figure 3). While the gas is spatially offset by $\sim 15 \mathrm{kpc}$, its velocity of $v_{h} \sim-500 \mathrm{~km} \mathrm{~s}^{-1}$ is remarkably close to that of the GSS at this point. While they may be unrelated, it is worthwhile to examining if it is plausible that they are physically connected. Such a question is complicated by the fact that there appears to be very little gas associated with the GSS, consistent with a lack of recent star formation (Brown et al. 2006), but this could be solved if the enhancement of H I marks the (as yet unidentified) progenitor of the GSS, with the offset in the spatial location illustrating the action of ram-pressure stripping; such a conclusion would indicate that the accretion of the GSS is a relatively recent event and has not undergone several complete orbits, or by a progenitor massive enough to retain some of its gas through a prolonged accretion. This latter option is in agreement with the prediction of Fardal et al. (2008) who suggested that the source of the GSS was a rotating disk galaxy with a stellar mass of $\sim 10^{9} M_{\odot}$.

An alternate explanation also requires that the progenitor of the GSS be gas-rich, but that some gas remain orbiting with the stars. This gas would be too tenuous to be visible in the observations presented in this paper, but as the stream is funneled into the central regions, orbits converge and the local densities of both stars and gas increase. Given this density enhancement, the gas could become visible to our observations. Again, the offset in spatial location probably indicates ongoing ram pressure, although there may be a delicate balance between the amount of ongoing ram pressure and the dynamical shepherding by the associated stream of stripped dark matter, so that any gas can remain associated with the stream. Detailed simulations of the formation and evolution of the GSS, considering realistic gas physics, are required to address these questions.

Away from M31, there is a tantalizing correlation between $\mathrm{H}$ I and stellar material in the vicinity of the SW Cloud. There are two potential interpretations for this, one with the SW Cloud representing an outwardly moving agglomeration of shredded stars, left over from a close interaction with M31 250 million years ago. This interaction would have to have been with a relatively pristine, gas-rich progenitor to leave the shredded debris with gas. On the other hand, the SW Cloud could represent the enhancement at the turning point of a stellar stream, and this stream would again have to be gas-rich to exhibit a similar enhancement in the $\mathrm{H}$ I. Both scenarios require a relatively recent first passage of M31, ensuring that the progenitor does not lose all its gas through ram-pressure stripping through an extended interaction. The estimated stellar and gas masses in the region of the SW Cloud are $\sim 10^{7} M_{\odot}$ and $\sim 5 \times 10^{5} M_{\odot}$, respectively, suggesting that if this is representative of a recent accretion event, then the progenitor would have properties similar to the dwarf irregular IC10 (McConnachie 2012).

While we do not have kinematics for the stellar component of the SW Cloud, it does appear to be spatially correlated with three globular clusters (see Mackey et al. 2010) for which velocities have been recently determined (these will be presented in detail in forthcoming publications by Mackey et al. 2012 and J. Veljanoski et al. 2013, in preparation). If we take their velocities, of $\sim-433 \mathrm{~km} \mathrm{~s}^{-1}$ (PAndAS-7), $\sim-411 \mathrm{~km} \mathrm{~s}^{-1}$ 
(PAndAS-8), and $\sim-363 \mathrm{~km} \mathrm{~s}^{-1}$ (PAndAS-14), each with an error of less than $10 \mathrm{~km} \mathrm{~s}^{-1}$, as being representative of that of the SW Cloud in general, we can compare this gaseous spur velocity of $\sim-470 \mathrm{~km} \mathrm{~s}^{-1}$, which is broadly consistent with the globular cluster velocity, especially if we expect a velocity gradient along any putative stream connecting the two components. It should be noted, however, that the width of the $\mathrm{H}$ I in the SW Spur is $\sim 200 \mathrm{~km} \mathrm{~s}^{-1}$, again indicating that we may be looking at both outbound and inbound gaseous streams, although until we have stellar kinematics for the SW Cloud and detailed dynamical modeling, the link between the SW Cloud and SW Spur remains circumstantial.

The distribution of gas within the M31-M33 system, especially the warped gas disk of M33 and the apparent gas bridge connecting M31 to M33, strongly suggests a past interaction of the two galaxies. It has been argued that the misalignment between the inner and outer gaseous disk of M33 and its interpretation as a warped disk could be the result of a recent tidal interaction (this is discussed in more detail below). The faint extension of the stellar disk with an alignment similar to the outer gas disk can be reproduced in interaction models (McConnachie et al. 2009). The gas bridge between the two galaxies has also been modeled in the context of a past interaction (e.g., Bekki 2008).

While the interaction hypothesis is a plausible explanation for the phenomenology of the gas in the M31-M33 system, it ultimately depends on knowing the orbit of M33. Recent measurements of both the proper motion of M33 (Brunthaler et al. 2005) and M31 (Sohn et al. 2012) provide strong constraints on the M33 orbit within assumed mass models of M31. We have been recently extending the dynamical models described in McConnachie et al. (2009) to describe the distorted outer stellar disk of M33 to a range of mass models for M31 and M33 using a Bayesian analysis to determine the distribution of orbits that are statistically consistent with the observed distance, radial velocities, and proper motions of the galaxies. This is a work in progress but we present some preliminary results here relevant to the observed gas distribution. In Figure 5, we present the distribution of orbits and the resulting line-ofsight velocity field consistent with the observations with the constraints that the pericenter for the M33 orbit is in the range of 30-60 kpc in a mass model of M31 with $M=2.5 \times 10^{12} M_{\odot}$ with $r_{200}=280 \mathrm{kpc}$. The orbits are computed within the model potentials including a Chandrasekhar drag term to model dynamical friction. The drag coefficients are calibrated against live $N$-body simulations to ensure their applicability to the Bayesian analysis. We further constrain the distribution to lie within $20^{\circ}$ of the plane containing the gas bridge with the implicit assumption that the bridge is created from tidally stripped gas from M33. We have also overlaid the stellar distribution from one $N$-body simulation including dark matter and stars without gas to orient the orbital distribution as well as illustrate the expected distortion of the outer disk of M33. We note that the complex stellar features seen around M33 in the simulation would be undetectable using current observations but we present an enhanced view to illustrate the complex dynamics. This model succeeds in two points with respect to the gas distribution. First, the model predicts that the outer disk of M33 will be warped and twisted as the result of an interaction while the inner bright disk will remain unscathed. Second, tidally stripped stars result in a tidal tail extending to the SE and a tidal bridge that falls back onto M31. While we have not modeled the gas in this simulation, we expect stripped gas to follow approximately

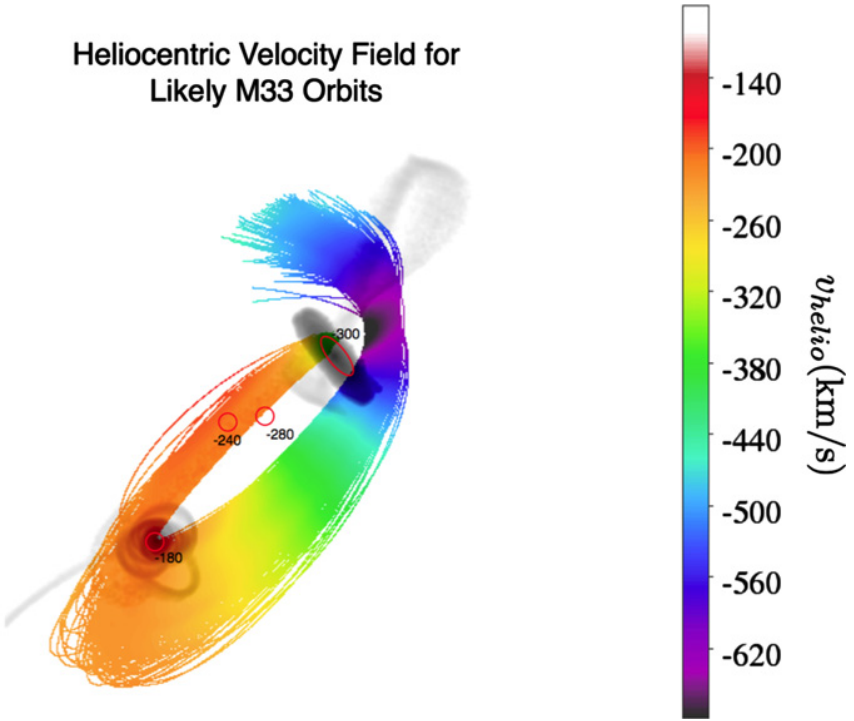

Figure 5. Heliocentric velocity field from a distribution of likely M33 orbits overlaid on an image from a simulation of a hypothetical interaction between M33 and M31. Also shown are the positions of some of the main gas features from Figure 2 and their observed line-of-sight velocities. The simulation contains only stars and dark matter without gas and shows an enhanced view of the distorted outer disk of M33 and tidally stripped stars. Some outer disk stars are thrown outward on a tidal tail to the SE while others form a tidal bridge back toward M31. The velocity field is generated from a distribution of orbits generated from a Bayesian analysis that are statistically consistent with the known distances, radial velocities, and proper motions of M31 and M33 and their given errors. The orbits are further constrained by the assumption that the M33 orbit passed within 30-60 kpc at pericenter assuming a mass model of M31 with $M=2.5 \times 10^{12} M_{\odot}$ and $r_{200}=280 \mathrm{kpc}$ with the plane of the orbit passing close to the "gas bridge" between M31 and M33. The close agreement between the velocity of density peaks on the gas bridge and orbital velocities of M33 in interacting models supports the idea that the bridge may be tidally stripped gas from M33 from a recent interaction. A caveat is that the pre-encounter gas disk of M33 would need to extend to $\sim 15 \mathrm{kpc}$.

(A color version of this figure is available in the online journal.)

ballistic trajectories similar to the stars. The heliocentric velocity of the distribution of orbits is consistent with the observed velocities of -240 and $-280 \mathrm{~km} \mathrm{~s}^{-1}$ seen in prominent gas features in the bridge. A tidal bridge of gas falling back onto M31 leading the position of M33 on its orbit after a past interaction is a plausible description of these features.

Finally, we look more closely at the stellar and gaseous material in the outskirts of M33, with distinct features visible in both the NW and SE (see Figure 4). The position angle of the $\mathrm{H}$ I feature is offset from that of the disk by $\sim 30^{\circ}-40^{\circ}$ and has been interpreted as a warped disk in projection and modeled in terms of tilted rings by Corbelli \& Schneider (1997). The stellar features lie roughly between the disk and $\mathrm{H}$ i position angles.

The origin of both the $\mathrm{HI}$ and stellar features has been attributed to tidal interactions with M31 during a close encounter (Putman et al. 2009; McConnachie et al. 2009; but see Reakes \& Newton 1978 for an early discussion of this possibility), though models of a steady state precessing warp in a model with a flattened halo have also been considered (e.g., Kuijken 1991). Competing with tidal effects is the gravitational field of the M33 disk and dark halo. In addition, the H I disk experiences ram pressure due to its interaction with the gaseous halo of M31. We can distinguish between three regions: the outermost part of the disk where tidal interactions and ram pressure strip material from M33 leaving behind a stream, which roughly traces the M33 orbit; an intermediate region, where material is stripped 


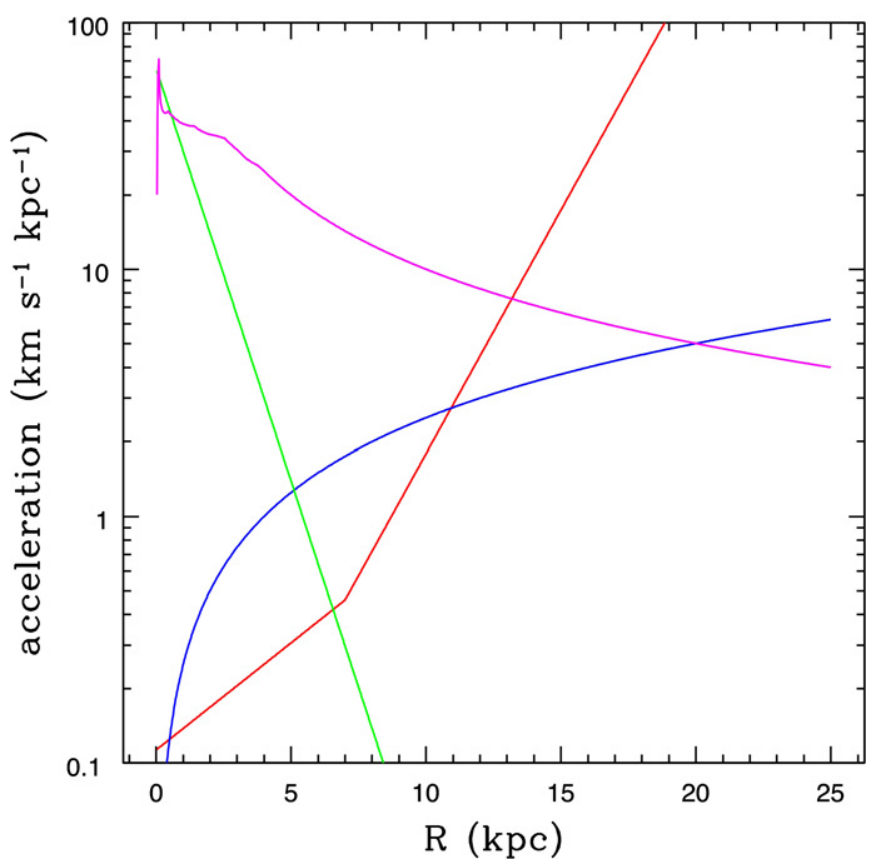

Figure 6. Various components of force (per unit mass) for the restoring force of M33 (magenta) and the disk (green), and ram pressure (red) and the M31 tidal field (blue). At the tidal radius, $R_{t} \simeq 20 \mathrm{kpc}$, the forces due to the M31 and M33 tidal fields are equal, while ram-pressure stripping can disrupt the gaseous disk at $R \gtrsim 7 \mathrm{kpc}$ and completely strip material from beyond $R \gtrsim 14 \mathrm{kpc}$.

(A color version of this figure is available in the online journal.)

from the M33 disk but remains bound to the galaxy; and a close-in region, where the disk is warped but material remains on roughly circular orbits.

In this simplified argument, we consider an element of the M33 gas disk at galactocentric radius $R$ with surface area $\Delta \Omega$ and surface density $\Sigma_{\text {gas }}$. The tidal force on this element due to M31 is

$$
F_{\text {tid }} \simeq \Sigma_{\text {gas }} \frac{V_{\mathrm{M} 31}^{2}}{R_{p}^{2}} R \Delta \Omega,
$$

where $R_{p}$ is the perigalactic distance between M31 and M33. In deriving this expression we have assumed that $R_{p} \gg R$ and that M31 has a flat rotation curve with a circular velocity $V_{\mathrm{M} 31}$. The ram-pressure force is

$$
F_{\text {rp }} \simeq \rho_{\text {gas }} V_{\text {rel }}^{2} \Delta \Omega
$$

where $\rho_{\text {gas }}$ is the density of the M31 gaseous halo. The gravitational restoring force due to the M33 disk is

$$
F_{d, \text { res }} \simeq 4 \pi G \Sigma_{\text {disk }} \Sigma_{\text {gas }} \Delta \Omega
$$

where $\Sigma_{\text {disk }}$ is the total surface density of the disk. If we assume an exponential disk, then $\Sigma_{\text {disk }}=\left(M_{d} / 4 \pi G R_{d}\right) \exp \left(-R / R_{d}\right)$. The restoring force due to M33 as a whole is

$$
F_{t, \text { res }}=\frac{V_{\mathrm{M} 33}^{2}}{R} \Sigma_{\text {gas }} \Delta \Omega,
$$

where we assume that M33 also has a flat rotation curve. Setting $F_{\text {tid }}=F_{t, \text { res }}$ yields an estimate for the tidal radius: $R_{t} \simeq R_{p}\left(V_{\mathrm{M} 33} / V_{\mathrm{M} 31}\right)$.

The different forces are shown in Figure 6, and for purely illustrative purposes, we set $V_{\mathrm{M} 31}=250 \mathrm{~km} \mathrm{~s}^{-1}, V_{\mathrm{M} 33}=$ $100 \mathrm{~km} \mathrm{~s}^{-1}, V_{\text {rel }}=400 \mathrm{~km} \mathrm{~s}^{-1}, R_{p}=50 \mathrm{kpc}, M_{d}=2.6 \times$
$10^{9} M_{\odot}, R_{d}=1.1 \mathrm{kpc}$, and $\rho_{\mathrm{gas}}=8 \times 10^{-5} \mathrm{~cm}^{-3} \mathrm{~m}_{H}$. The forces due to the M31 tidal field and due to M33 itself are equal at the tidal radius $R_{t} \simeq 20 \mathrm{kpc}$. Ram pressure can disrupt the disk at $R \gtrsim 7 \mathrm{kpc}$ and strip material from M33 at $R \gtrsim 14 \mathrm{kpc}$.

While these arguments represent order-of-magnitude estimates, they appear to roughly correspond to the observed structures in M33. However, a full exploration of the M31-M33 interaction, especially with regards to the stripping of M33, using models including gas, stars, and dark matter, are required to fully understand the complex dynamics on display; this will form the basis of an upcoming paper (J. Dubinski et al. 2013, in preparation).

\section{CONCLUSIONS}

We have presented the spatial and kinematic correlations of the stellar and gaseous substructures within the halos of M31 and M33. While significant substructure is apparent in each component, and there is a gross alignment of significant features, mainly along the axis connecting M31 and M33, there is a distinct lack of correlation between the detailed structures.

The lack of a truly global kinematic survey of the stellar substructure within the halos of M31 and M33 limits a detailed comparison of stars and gaseous material, although the growing number of "key-hole" observations with $10 \mathrm{~m}$ class telescopes is addressing this. However, this situation will be resolved with the advent of wide-field spectroscopy on large telescopes, such as the proposal to build the ngCFHT, ${ }^{19}$ ushering in a new era in understanding galactic archaeology.

The resulting conclusion of this study, therefore, is that the gaseous and stellar substructures within the halos of M31 and M33 have been built through a number of distinct accretion events, but differing physical processes have driven the disruption of the stellar and gaseous components of any particular substructure, with the latter suffering the additional forces of shocking and ram-pressure stripping. Given that, through the use of high-resolution numerical simulations, our understanding of the differing processes driving the disruption of stellar and gaseous material, a comparison of their distribution through the halo should allow us to dynamically date the accretion; this will be the subject of a future contribution.

G.F.L. gratefully acknowledges financial support for his ARC Future Fellowship (FT100100268) and through the award of an ARC Discovery Project (DP110100678). Furthermore, G.F.L. thanks the Institute of Astronomy at the University of Cambridge for their support and hospitality during his sabbatical during the latter half of 2010, where a substantial portion of this work was undertaken.

\section{REFERENCES}

Abraham, R. G., \& van den Bergh, S. 2001, Sci, 293, 1273

Bekki, K. 2008, MNRAS, 390, L24

Besla, G., Kallivayalil, N., Hernquist, L., et al. 2010, ApJL, 721, 97

Braun, R., \& Thilker, D. A. 2004, A\&A, 417, 421

Braun, R., Thilker, D. A., Walterbos, R. A. M., \& Corbelli, E. 2009, ApJ, 695,937

Brown, T. M., Smith, E., Ferguson, H. C., et al. 2006, ApJ, 652, 323

Brunthaler, A., Reid, M. J., Falcke, H., Greenhill, L. J., \& Henkel, C. 2005, Sci, 307, 1440

Bullock, J. S., \& Johnston, K. V. 2005, ApJ, 635, 931

Burton, W. B., \& Lockman, F. J. 1999, A\&A, 349, 7

Carlberg, R. G., Richer, H. B., McConnachie, A. W., et al. 2011, ApJ, 731, 124

19 http://orca.phys.uvic.ca/ pcote/ngcfht 
Chapman, S. C., Ibata, R., Irwin, M., et al. 2008, MNRAS, 390, 1437 Cockcroft, R., Harris, W. E., Ferguson, A. M. N., et al. 2011, ApJ, 730, 112 Collins, M. L. M., Chapman, S. C., Irwin, M., et al. 2009, MNRAS, 396, 1619 Collins, M. L. M., Chapman, S. C., Rich, R. M., et al. 2011, MNRAS, 417, 1170 Conn, A. R., Ibata, R. A., Lewis, G. F., et al. 2012, ApJ, 758, 11 Conn, A. R., Lewis, G. F., Ibata, R. A., et al. 2011, ApJ, 740, 69 Cooper, A. P., Cole, S., Frenk, C. S., et al. 2010, MNRAS, 406, 744 Corbelli, E. 2003, MNRAS, 342, 199

Corbelli, E., \& Schneider, S. E. 1997, ApJ, 479, 244

de Jong, J. T. A., Yanny, B., Rix, H.-W., et al. 2010, ApJ, 714, 663

Diaz, J., \& Bekki, K. 2011, PASA, 28, 117

Faber, S. M., Phillips, A. C., Kibrick, R. I., et al. 2003, Proc. SPIE, 4841, 1657

Fardal, M. A., Babul, A., Guhathakurta, P., Gilbert, K. M., \& Dodge, C. 2008, ApJL, 682, 33

Fardal, M. A., Guhathakurta, P., Gilbert, K. M., et al. 2012, MNRAS, 423, 3134

Faria, D., Johnson, R. A., Ferguson, A. M. N., et al. 2007, AJ, 133, 1275

Ferguson, A. M. N., Irwin, M. J., Ibata, R. A., Lewis, G. F., \& Tanvir, N. R. 2002, AJ, 124, 1452

Font, A. S., McCarthy, I. G., Crain, R. A., et al. 2011, MNRAS, 416, 2802

Geehan, J. J., Fardal, M. A., Babul, A., \& Guhathakurta, P. 2006, MNRAS, 366, 996

Gilbert, K. M., Guhathakurta, P., Beaton, R. L., et al. 2012, ApJ, 760, 76

Gilbert, K. M., Guhathakurta, P., Kollipara, P., et al. 2009, ApJ, 705, 1275

Gratier, P., Braine, J., Rodriguez-Fernandez, N. J., et al. 2010, A\&A, 522, A3

Huxor, A. P., Ferguson, A. M. N., Tanvir, N. R., et al. 2011, MNRAS, 414, 770

Huxor, A. P., Tanvir, N. R., Ferguson, A. M. N., et al. 2008, MNRAS, 385, 1989

Huxor, A. P., Tanvir, N. R., Irwin, M. J., et al. 2005, MNRAS, 360, 1007

Ibata, R., Chapman, S., Ferguson, A. M. N., et al. 2004, MNRAS, 351, 117

Ibata, R., Chapman, S., Ferguson, A. M. N., et al. 2005, ApJ, 634, 287

Ibata, R., Irwin, M., Lewis, G., Ferguson, A. M. N., \& Tanvir, N. 2001, Natur, 412, 49

Ibata, R., Martin, N. F., Irwin, M., et al. 2007, ApJ, 671, 1591

Ibata, R. A., \& Lewis, G. F. 1998, ApJ, 500, 575

Kalirai, J. S., Beaton, R. L., Geha, M. C., et al. 2010, ApJ, 711, 671

Kalirai, J. S., Zucker, D. B., Guhathakurta, P., et al. 2009, ApJ, 705, 1043

Klypin, A., Kravtsov, A. V., Valenzuela, O., \& Prada, F. 1999, ApJ, 522, 82

Kuijken, K. 1991, ApJ, 376, 467

Mackey, A. D., Huxor, A., Ferguson, A. M. N., et al. 2006, ApJL, 653, 105

Mackey, A. D., Huxor, A., Ferguson, A. M. N., et al. 2007, ApJL, 655, 85

Mackey, A. D., Huxor, A. P., Ferguson, A. M. N., et al. 2010, ApJL, 717, 11
Mackey, A. D., Huxor, A. P., Ferguson, A. M. N., et al. 2012, MNRAS, in press (arXiv:1211.1103)

Martin, N. F., Ibata, R. A., Chapman, S. C., Irwin, M., \& Lewis, G. F. 2007, MNRAS, 380, 281

Martin, N. F., Ibata, R. A., Irwin, M. J., et al. 2006, MNRAS, 371, 1983

Mastropietro, C., Burkert, A., \& Moore, B. 2009, MNRAS, 399, 2004

McClure-Griffiths, N. M., Pisano, D. J., Calabretta, M. R., et al. 2009, ApJS, 181,398

McConnachie, A. W. 2012, AJ, 144, 4

McConnachie, A. W., Chapman, S. C., Ibata, R. A., et al. 2006, ApJL, 647, 25

McConnachie, A. W., Ferguson, A. M. N., Irwin, M. J., et al. 2010, ApJ, 723, 1038

McConnachie, A. W., Irwin, M. J., Ibata, R. A., et al. 2003, MNRAS, 343,1335

McConnachie, A. W., Irwin, M. J., Ibata, R. A., et al. 2009, Natur, 461, 66

Moore, B., \& Davis, M. 1994, MNRAS, 270, 209

Moore, B., Ghigna, S., Governato, F., et al. 1999, ApJL, 524, 19

Murakami, I., \& Babul, A. 1999, MNRAS, 309, 161

Putman, M. E., Peek, J. E. G., Muratov, A., et al. 2009, ApJ, 703, 1486

Putman, M. E., Thom, C., Gibson, B. K., \& Staveley-Smith, L. 2004, ApJL, 603, 77

Reakes, M. L., \& Newton, K. 1978, MNRAS, 185, 277

Richardson, J. C., Ferguson, A. M. N., Johnson, R. A., et al. 2008, AJ, 135,1998

Richardson, J. C., Irwin, M. J., McConnachie, A. W., et al. 2011, ApJ, 732, 76

Ruszkowski, M., Brüggen, M., Lee, D., \& Shin, M.-S. 2012, ApJ, submitted (arXiv:1203.1343)

Siebert, A., Williams, M. E. K., Siviero, A., et al. 2011, AJ, 141, 187

Sohn, S. T., Anderson, J., \& van der Marel, R. P. 2012, ApJ, 753, 7

Stanimirović, S., Hoffman, S., Heiles, C., et al. 2008, ApJ, 680, 276

Tanvir, N. R., Mackey, A. D., Ferguson, A. M. N., et al. 2012, MNRAS, 422, 162

Thilker, D. A., Braun, R., \& Walterbos, R. A. M. 2002, in ASP Conf. Proc. 276, Seeing Through the Dust: The Detection of HI and the Exploration of the ISM in Galaxies, ed. A. R. Taylor, T. L. Landecker, \& A. G. Willis (San Francisco, CA: ASP), 370

Thilker, D. A., Braun, R., Walterbos, R. A. M., et al. 2004, ApJL, 601, 39

Tolstoy, E. 2011, Sci, 333, 176

Tolstoy, E., Hill, V., \& Tosi, M. 2009, ARA\&A, 47, 371

Wyse, R. F. G. 2010, AN, 331, 526 